\title{
Energy conversion evolution at lunar polar sites
}

\author{
JAMES D BURKE \\ The Planetary Society, 65 N. Catalina Ave., Pasadena, CA 91106, USA. \\ e-mail: jdburke@its.caltech.edu
}

Lunar polar environments have many advantages from the standpoint of energy supply to robotic and human surface bases. Sunlight is nearly continuous and always horizontal at peaks of perpetual light, while waste heat rejection is aided by the existence of cold, permanently shadowed regions nearby. In this paper a possible evolution of lunar polar energy systems will be described, beginning with small robotic photovoltaic landers and continuing into the development of increasingly powerful and diverse energy installations to provide not only electric power but also piped-in sunlight, air conditioning and high-temperature process heat.

\section{Introduction}

In the seventeenth century, J D Cassini, the first director of the Paris Observatory, accurately described the motions of the Moon, including the orientation and precession of its spin axis relative to its orbit and the plane of the ecliptic. The Moon's polar axis is inclined only about oneand-a-half degrees from the ecliptic pole, with the result that sunlight is nearly continuous and always horizontal in lunar polar regions.

Among the consequences of this are the existence of permanent shadow in some crater bottoms and the existence of almost uninterrupted sunlight at some high peaks along crater rims (Bussey et al 1999). The polar thermal environment is thus much more favorable for surface operations, both robotic and human, than elsewhere on the Moon where surface systems must cope with two-week hot days and two-week frigid nights.

This paper is concerned with ways to take advantage of the situation near the lunar poles, emphasizing the energy conversion systems that are essential for surface activities and also touching on prospects for the use of local natural resources.

\section{Ideas about lunar polar resources and their uses}

In 1961 Murray, Watson and Brown analyzed the likely behavior of volatiles at the surface of the Moon and concluded that atoms and molecules, hopping around in the collisionless atmosphere, might become cold-trapped in the polar permanently dark regions. In subsequent years Arnold (1979) and others made additional predictions, but it was not until 1994-98 that observational data began to show possible cold-trapping near the poles. The most persuasive evidence came from neutron detectors on the Lunar Prospector Orbiter (Nozette et al 2001), showing an excess of light atoms, most likely hydrogen, that many people immediately interpreted as due to the presence of water ice within a few meters of the surface. However, the presence or absence of ice, their form and their utility, if any, remain to be demonstrated by in situ observation.

Meanwhile the present author had been advocating the use of polar regions, with or without the putative ice resource, as preferred base locations (Burke 1978, 1995) primarily because of their more favorable thermal environments. Now this prospect

Keywords. Energy generation; solar energy; electric power; lunar environment. 
is to be more thoroughly investigated in the coming lunar orbital missions. As a result, this is a good time for considering polar surface activities, both robotic and human, that should occur in the coming years.

\section{Desired properties of polar energy systems}

Landing precisely at a known near-polar location is a goal for the next stage of lunar technology development. Improved topographic and selenodetic mapping, as planned in several coming lunar polar orbital missions, will help to make this possible. Assuming that a Surveyor-class robotic lander can be placed atop a peak of perpetual light, an obvious technology goal for the mission is to demonstrate continuous solar photovoltaic power. One way to do this is to provide a vertical-axis, cylindrical solar array as described in Burke and Dokbua (2004), where an array like that of the Boeing/Hughes HS 378 communication satellite series is envisioned. Probably the thermal design of the lunar solar power collector would have to be different from that of the spinning satellite because of the Moon's very slow rotation, but it should still be possible to obtain several hundred watts of electric power with an array-plus-structure mass of less than $100 \mathrm{~kg}$.

Power from such a collector would not be truly uninterrupted, both because of terrain shadowing as described in Bussey et al (1999) and because of lunar eclipses. Therefore, the power-system design must include battery energy, just as is done to deal with the eclipses that occur in geosychronous orbit.

The next logical step after a fixed, cylindrical photovoltaic collector is a sun-tracking array. As seen from a lunar pole, the Sun moves around on the horizon at a rate of about 1/2 degree per hour. In Burke (1978) a rotating power tower is imagined, combining photovoltaic and heat-delivery elements for base environmental control and hightemperature material processing. For the lunar base concepts described by McKay et al (1992) one more function is added; namely, piped-in sunlight for illumination, interior lighting and photosynthesis.

\section{A possible evolution}

After the current generation of robotic lunar orbiters (SMART-1, Lunar-A, SELENE, Chandrayaan-1, Chang'E-1 and a possible US Lunar Reconnaissance Orbiter) has provided accurate data on lunar polar landforms, it will be possible to make precision landings using any of a variety of techniques beginning with en route navigation and ending with local map-matching. Many years ago, a human-piloted version of this sequence was demonstrated when the Apollo 12 crew landed less than 170 meters from Surveyor 3 (figure 1). That feat was achieved despite the absence of precise selenodesy because the location of the Surveyor was very accurately known from radio tracking of it after landing. The Apollo crew, guided to Surveyor's vicinity by a combination of inertial, celestial and radio references, then made their final approach using landmarks as imaged by lunar orbiters.

The first polar mountain top lander should have, in addition to precise post-landing radio location and the photovoltaic cylinder mentioned above, cameras for observing the local scene and perhaps also deployed cameras for observing itself, plus power monitors and other housekeeping instrumentation. Should it also have some limited mobility? This would be a good addition if only for leveling and erection of tall inflatable elements, as desired if the mission were to include those as engineering precursors for the payloads of larger later landers. One such application, in the form of an art object, is discussed by Burke and Dokbua (2004). Of course, science instruments would also be accommodated. Continuous observation of particles and fields, including electrostatically-levitated dust, immediately above the lunar surface would be a good goal both for science and for engineering evaluation of the polar surface environment.

Either contemporary with this first powergenerating lander or closely following it, a robotic rover should land in or travel to a dark region to find out the real meaning of the excess polar hydrogen. Energy storage and conversion for this class of rovers presents interesting tradeoffs. Since the machine must operate in darkness and out of sight from Earth, it must use batteries, fuel cells, radioactive sources or a combination of these for power and be largely autonomous. Microwave beaming from an illuminated site is a more speculative option. The observed neutron energies have been interpreted as showing hydrogen, and possibly ice, within a meter or two of the surface. The rover should be able to drill at least to this depth. That, in addition to its mobility and other subsystem needs, implies a peak power demand of at least a few hundred watts.

Following the first mountain top lander and dark-region rover, a program of precursor technology and operation demonstration should proceed. In July-August 2004, a project team at the International Space University's session in Adelaide, Australia examined this prospect (ISU Project 2004). Among their recommendations were robotic evaluation of resource acquisition and processing, 


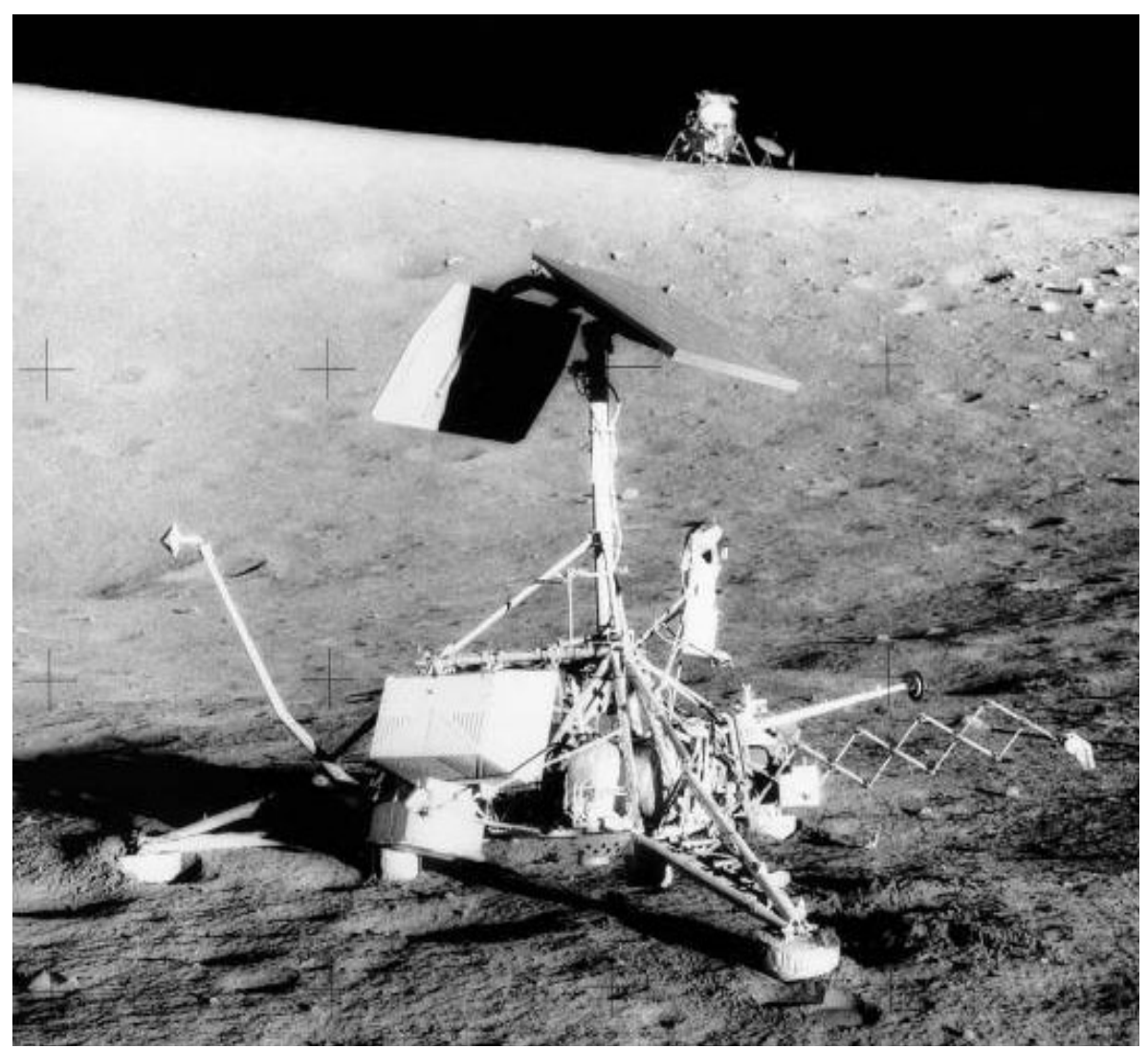

Figure 1. Surveyor 3 in 1969 after 31 months on the Moon. Apollo 12 lunar module in background.

followed first by human missions lasting a few weeks and then by missions lasting many months, modeling the human challenges of flight to and from Mars.

To enable this sequence it will be necessary to establish steadily-growing capabilities at a polar base site. One essential requirement will be excavation and moving of regolith, for example to pile lunar material on top of a habitat for radiation shielding and thermal control. Bringing solarderived energy into base interiors presents a variety of design challenges. Photovoltaic electricity is the simplest, but its delivery implies a need for efficient waste-heat rejection, for example by means of radiators in dark regions. Direct solar lighting, heating and air conditioning will require passages like periscopes that can admit light and heat photons, as described by McKay et al (1992) while excluding ionizing radiation.

\section{Conclusion}

With a sun-tracking power tower, underground energy-user facilities and waste-heat rejection to dark space, a polar lunar base could ultimately become the prototype for the energy-conversion systems that will be needed when humans step up to the challenge of permanent inhabitation of the Moon.

\section{References}

Arnold J R 1979 Ice in the lunar poles; J. Geophys. Res. 84 5659-5668.

Burke J D 1978 Energy conversion at a lunar polar site; In: Radiation Energy Conversion in Space (eds) Kenneth W Billman and Martin Summerfield; Progress in Astronautics and Aeronautics 61, AIAA, New York.

Burke J D 1995 Merits of a lunar polar base location; In: Lunar Bases and Space Activities of the 21st Century (ed.) Mendell W W, Lunar and Planetary Institute, Houston.

Burke J D and Dokbua R 2004 Practical opportunities for including art in coming lunar programs; 7th ESTEC workshop on space and the arts, Noordwijk.

Bussey D B J, Spudis P D and Robinson M S 1999 Illumination conditions at the lunar south pole; Geophys. Res. Lett. 26(9) 1187-1190.

McKay M F, McKay D S and Duke M B (eds) 1992 Space Resources (4 vols.) NASA SP-509.

Nozette S et al 2001 Integration of remote-sensing data sets: Evidence for ice at the lunar south pole; J. Geophys. Res. 106, E19, 23,253-23,266.

Students of ISU 2004 LunAres: International Lunar Exploration in Preparation for Mars; International Space University, Strasbourg.

Watson K, Murray B C and Brown H 1961 The behavior of volatiles on the lunar surface. J. Geophys. Res. 66 3033-3040. 